\title{
ERRATA
}

\section{A PARALLEL WILF ALGORITHM FOR COMPLEX ZEROS OF A POLYNOMIAL}

\author{
E. V. KRISHNAMURTHY and H. VENKATESWARAN
}

The procedure given in BIT 21,1 should read:

procedure BISECTION; \{Input to this procedure: The rectangle $R$, the tolerance eps, number of zeros within $R\}$;

begin $_{1}$ If side of $R>$ eps then begin $_{2}$ Subdivide as Left/Right or Top/Bottom;

\{approximately - say $R 1$ and $R 2$ \};

Trace zeros, if any, on the dividing line; $\{$ say $m\}$;

If $m<n$ then

begin $_{3}$ Count zeros in $R 1$; $\{$ say $N R 1\} ; N R 2:=(n-m)-N R 1$

If $R 1$ has all the zeros then

$\operatorname{BISECTION}(R 1$, eps, $N R 1)$

else If $R 2$ has all the zeros, then

BISECTION $(R 2$, eps, $N R 2)$

else $\quad \operatorname{BISECTION}(R 1$, eps, $N R 1)$

and $\operatorname{BISECTION}(R 2$, eps, NR2)

end 2

end 3

else the $n$ zeros $:=$ the centre of $R$

end 1

end BISECTION;

Note: 1. The connective and denotes that the two parts can go in parallel.

2. At the start of this procedure the input is an initial square containing all the zeros of $P(z)$. 